ARTICLE

\title{
A multi-responsive healable supercapacitor
}

\author{
Haili Qin ${ }^{1}$, Ping Liu ${ }^{1}$, Chuanrui Chen ${ }^{1}$, Huai-Ping Cong ${ }^{1 凶} \&$ Shu-Hong Yu (i) ${ }^{2 凶}$
}

Self-healability is essential for supercapacitors to improve their reliability and lifespan when powering the electronics. However, the lack of a universal healing mechanism leads to low capacitive performance and unsatisfactory intelligence. Here, we demonstrate a multiresponsive healable supercapacitor with integrated configuration assembled from magnetic $\mathrm{Fe}_{3} \mathrm{O}_{4} @ \mathrm{Au}$ /polyacrylamide (MFP) hydrogel-based electrodes and electrolyte and Ag nanowire films as current collectors. Beside a high mechanical strength, MFP hydrogel exhibits fast optical and magnetic healing properties arising from distinct photothermal and magnetothermal triggered interfacial reconstructions. By growing electroactive polypyrrole nanoparticles into MFP framework as electrodes, the assembled supercapacitor exhibits triplyresponsive healing performance under optical, electrical and magnetic stimuli. Notably, the device delivers a highest areal capacitance of $1264 \mathrm{mF} \mathrm{cm}-2$ among the reported healable supercapacitors and restores $~ 90 \%$ of initial capacitances over ten healing cycles. These prominent performance advantages along with the facile device-assembly method make this emerging supercapacitor highly potential in the next-generation electronics.

\footnotetext{
${ }^{1}$ Anhui Province Key Laboratory of Advanced Catalytic Materials and Reaction Engineering, School of Chemistry and Chemical Engineering, Hefei University of Technology, Hefei, P. R. China. ${ }^{2}$ Division of Nanomaterials and Chemistry, Hefei National Laboratory for Physical Sciences at Microscale, Institute of Energy, Hefei Comprehensive National Science Center, Department of Chemistry, Institute of Biomimetic Materials \& Chemistry, University of Science and

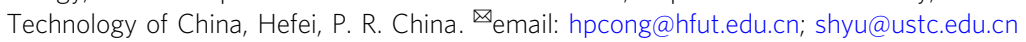


S elf-healing ability, widely found in biological tissues, is an attractive feature to repair internal or external damages automatically and allow structural and functional restorations, which is tightly related to long lifetime, good sustainability and high utilizing safety of artificial materials ${ }^{1-3}$. With the rapid development of wearable energy-storage devices, smart supercapacitors with self-healability have attracted particular research interests as they can restore their capacitive performance in the case of mechanical and structural damages under bending or other deformations. Most of the reported healable supercapacitors have been fabricated by either employing an extra selfhealing polymer layer to wrap/support the electrode ${ }^{4-7}$, or using an additional electrode patch combined with the self-recovered electrolyte $^{8}$. These non-intrinsically self-healing configurations between two electrodes sandwiched with an indispensable electrolyte layer lead to unsatisfactory healing efficiency and low energy density. Therefore, it is of great practical significance to realize and maintain integrated current collector-electrodeelectrolyte-electrode-current collector configuration during the device fabrication and after mechanical fractures ${ }^{9}$. Facing with these challenges, the creation of effective healing motifs for design and construction of an omni-healable supercapacitor is highly desirable and important.

Until now, various healing mechanisms have been exploited to fabricate self-healing materials, including incorporation/release of curing agents ${ }^{1}$, and introduction of dynamic/reversible covalent or noncovalent interactions as the mobile phases ${ }^{2,10-13}$. Among them, dynamic metal-ligand coordination interactions have demonstrated their effectiveness in constructing smart materials with the repeated self-healing capability and enhanced mechanical property simultaneously because of their modest binding strength and adjustable stimuli-responsive thermodynamic equilibrium ${ }^{14-16}$. Recently, we have reported the synthesis of robust and smart nanocomposite hydrogels with great selfhealing capability based on reversible gold-thiolate (Au-SR) coordination chemistry under the stimulus of near-infrared (NIR) laser ${ }^{17}$. Compared with thermally healable systems, the stimulus of light is undamaged to materials through exclusively exposing and healing the fractured region ${ }^{14,18}$. Moreover, photothermal property of noble metal can dissociate the metal-ligand motifs effectively, achieve surface reconstruction of coordination bonds, and therefore heal the fractures.

Meanwhile, magneto-thermal behavior, a fascinating origin of heat under a magnetic field, has been intensely studied in magnetic hydrogels composed of magnetic nano- or microparticles within the polymer hydrogel matrix ${ }^{19-22}$. So far, significant progress has been mainly made in the bio-fields of remotely controllable drug release and hyperthermal therapy ${ }^{21,23,24}$. In contrast, the magneto-thermal induced healing motifs have not been reported, although it is well accepted as a remotely controllable, homogeneous, and undamaged stimulus. One challenge is involved in achieving uniform distribution of magnetic nanoparticles in the hydrogel and preventing them from diffusing out of the matrices. Intelligently developing magneto-thermal triggered healing mechanism rather than the simple magnetic motion also remains great challenging $5,25,26$.

Herein, a kind of magnetic $\mathrm{Fe}_{3} \mathrm{O}_{4} @ \mathrm{Au}$ /polyacrylamide (PAM) (MFP) hydrogel has been fabricated by chemically crosslinking disulfide bond-functionalized $\mathrm{Fe}_{3} \mathrm{O}_{4} @ \mathrm{Au}$ nanocomposites into the polymeric network. Owing to effective energy dissipating mechanism from the homogeneously interconnected network structure, the MFP hydrogel demonstrates tough mechanical performance with large stretchability of $2250 \%$ its initial length and strong notch-insensitivity. Additionally, notable photothermal and magneto-thermal properties of the hydrogel enable the fabricated hydrogel with fast optical and remotely controllable magnetic healability relied on high-density dynamic $\mathrm{Au}-\mathrm{SR}$ coordination bonds. Incorporating polypyrrole (PPy) nanoparticles into the MFP network as electrode, a supercapacitor prototype is assembled by sandwiching two MFP-PPy electrodes with a MFP hydrogel electrolyte and spray-coating silver nanowire (AgNW) films as current collectors. The synergistic effects of $\mathrm{Au}, \mathrm{Ag}-\mathrm{SR}$ bonds allow the assembled supercapacitor with integrated configuration to exhibit the largest areal capacitance up to $1264 \mathrm{mF} \mathrm{cm}^{-2}$ and a record-breaking device-level stretchability of $1200 \%$, confirming it as one of the best performers among the flexible and healable supercapacitors. Impressively, this supercapacitor possesses intrinsically multi-responsive healing capability with $\sim 90 \%$ of capacitance restored over 10 optical, electrical, and magnetic healing cycles, respectively. These great performances and simple device-assembly method promise the presented supercapacitors highly competitive in the nextgeneration wearable and portable electronic devices.

\section{Results}

Design and preparation of magnetic hydrogel. Figure 1a shows schematic illustrations of the preparation of MFP hydrogel. Typically, $\mathrm{Fe}_{3} \mathrm{O}_{4} @ \mathrm{Au}$ composites were prepared through in situ reduction of $\mathrm{Au}$ nanoparticles on hydrothermally-synthesized $\mathrm{Fe}_{3} \mathrm{O}_{4}$ nanospheres ${ }^{27}$ at room temperature. The pre-obtained $\mathrm{Fe}_{3} \mathrm{O}_{4}$ nanospheres with a uniform diameter of $\sim 200 \mathrm{~nm}$ were monodispersed and consisted of small nanoparticles with a size of $15 \mathrm{~nm}$, behaving fast magnetic response when closed to a magnet (Supplementary Fig. 1a-d). Magnetic characterization indicated the superparamagnetism of $\mathrm{Fe}_{3} \mathrm{O}_{4}$ nanospheres with magnetization saturation value of $72.1 \mathrm{emu} \mathrm{g}^{-1}$ (Supplementary Fig. 2), confirming its nanoparticle-composed structure. With mild chemical reduction, the $\mathrm{Fe}_{3} \mathrm{O}_{4}$ nanospheres were decorated with large amount of $\mathrm{Au}$ nanoparticles with uniform size of $\sim 5 \mathrm{~nm}$, maintaining a monodispersed state (Supplementary Fig. 1e-h). X-ray diffraction (XRD) pattern and survey X-ray photoelectron spectroscopy (XPS) spectrum as well as core-leveled Au $4 f$ XPS spectrum of $\mathrm{Fe}_{3} \mathrm{O}_{4} @ \mathrm{Au}$ composites proved the anchoring of $\mathrm{Au}$ nanoparticles onto $\mathrm{Fe}_{3} \mathrm{O}_{4}$ nanospheres (Supplementary Figs. 3 and 4). Notably, the obtained $\mathrm{Fe}_{3} \mathrm{O}_{4} @ \mathrm{Au}$ composites held superparamagnetic characteristics with similar magnetization saturation value to $\mathrm{Fe}_{3} \mathrm{O}_{4}$ nanospheres, as shown from no remanence detected in the corresponding magnetic hysteresis loop (Supplementary Fig. 2). In order to get a kind of crosslinker for the hydrogel polymerization, the disulfide bond-ended $\mathrm{N}, \mathrm{N}^{\prime}$-bis (acryloyl)cystamine (BACA) molecules were used and linked with $\mathrm{Fe}_{3} \mathrm{O}_{4} @ \mathrm{Au}$ nanospheres via the $\mathrm{Au}-\mathrm{SR}$ bonds, producing the $\mathrm{Fe}_{3} \mathrm{O}_{4} @ \mathrm{Au} @ \mathrm{BACA}$ composites. As shown in transmission electron microscopy (TEM) image (Fig. 1b) and corresponding elemental mappings (Fig. 1c-e), the elements of Fe, $\mathrm{Au}$, and $\mathrm{S}$ were homogeneously distributed in the $\mathrm{Fe}_{3} \mathrm{O}_{4} @ \mathrm{Au} @ \mathrm{BACA}$ composites, demonstrating the success in binding BACA onto $\mathrm{Fe}_{3} \mathrm{O}_{4} @ \mathrm{Au}$. Subsequently, by serving $\mathrm{Fe}_{3} \mathrm{O}_{4} @ \mathrm{Au} @ \mathrm{BACA}$ composites as the crosslinkers, the MFP hydrogel was fabricated through the freeradical polymerization of acrylamide monomer initiated by potassium persulfate (KPS) and accelerated by $\mathrm{N}, \mathrm{N}, \mathrm{N}^{\prime}, \mathrm{N}^{\prime}$-tetramethylethylenediamine (TEMED). Scanning electron microscopy (SEM) image showed that the MFP hydrogel delivered homogeneous network structure with the pore size of $\sim 1 \mu \mathrm{m}$ (Fig. 1f). The MFP hydrogel presented distinct superparamagnetism in the magnetic hysteresis loop and performed remarkable movement to the applied magnetic field (Fig. 1g), inheriting from the homogeneously dispersed $\mathrm{Fe}_{3} \mathrm{O}_{4} @ \mathrm{Au}$ composites. For comparison, the conventional chemically crosslinked polymeric hydrogel was prepared by using BACA as chemical crosslinker without addition of $\mathrm{Fe}_{3} \mathrm{O}_{4} @ \mathrm{Au}$ composites, denoted as CCP hydrogel. Because of 
a

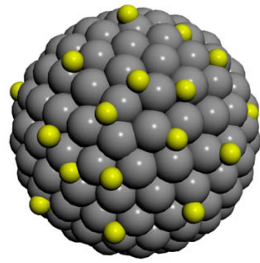

Fe3O4@Au

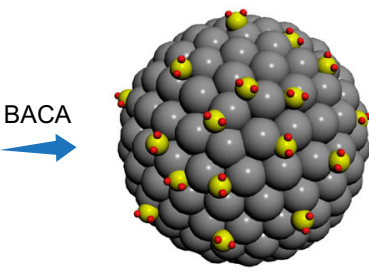

Fe3O4@Au@BACA crosslinker

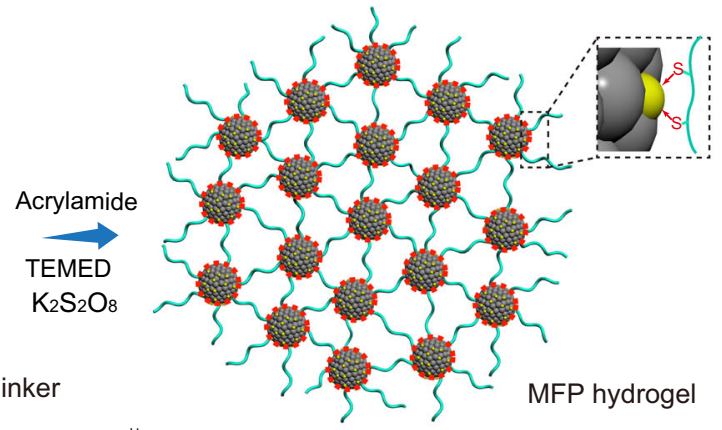

Au nanoparticle<smiles>CCC(C)C(=O)N(C)CCSCCCCC(N)N(C)CCO</smiles>

PAM chain
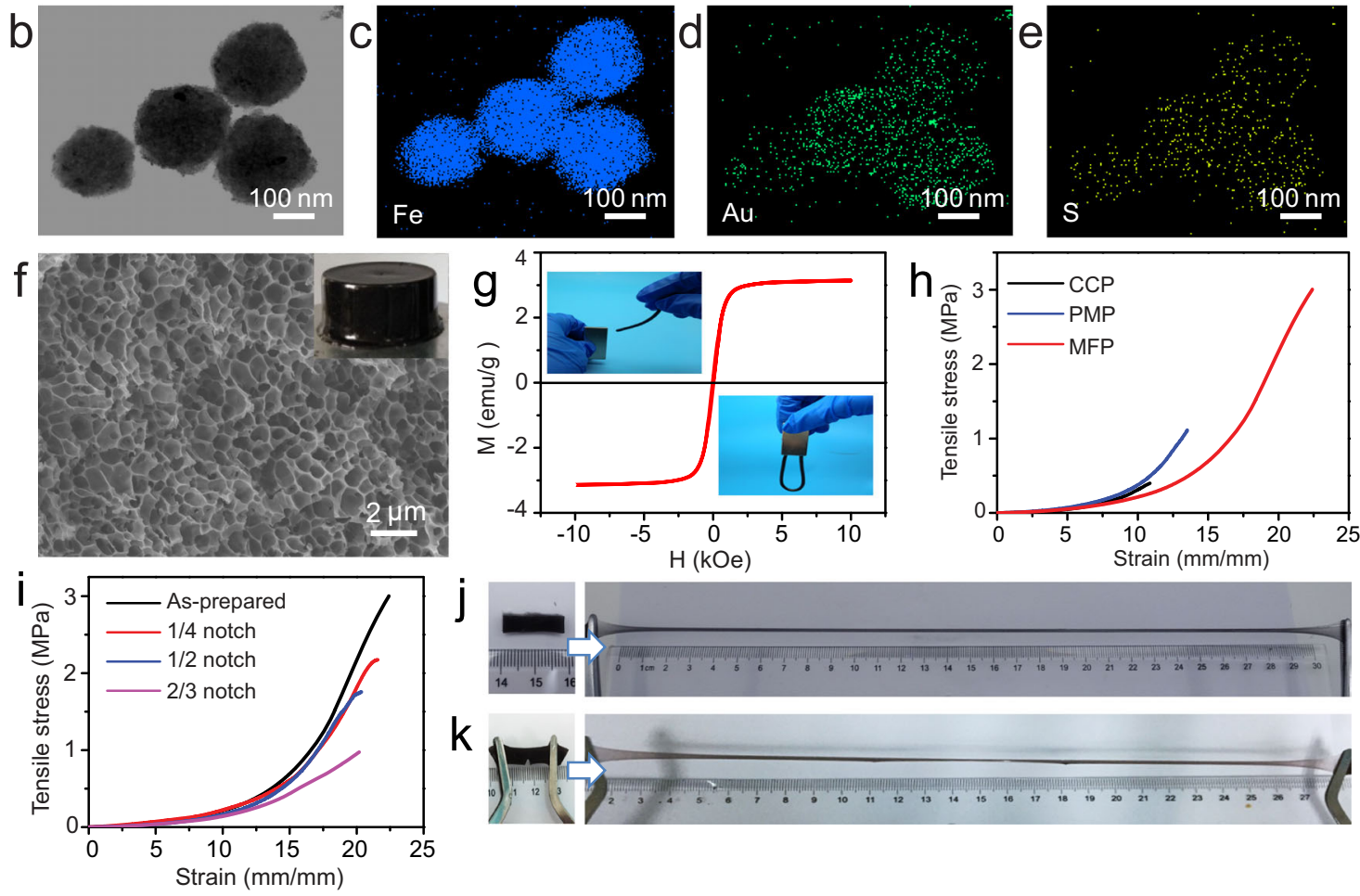

Fig. 1 Preparation and characterization of MFP hydrogel. a Schematic illustrations of the preparation of MFP hydrogel. $\mathbf{b}$ TEM image of $\mathrm{Fe}_{3} \mathrm{O}_{4} @ \mathrm{Au} @ \mathrm{BACA}$ composites. c-e Corresponding element mappings of Fe, Au, and S, suggesting the ultrafine coating of BACA. f SEM image of MFP hydrogel. The inserted photograph shows a columnar hydrogel. $\mathbf{g}$ Magnetic hysteresis loops of MFP hydrogel. The inserted optical images show magnetic response of MFP hydrogel with a magnet. $\mathbf{h}$ Tensile stress-strain curves of CCP, PMP, and MFP hydrogels. $\mathbf{i}$ Tensile stress-strain curves of MFP hydrogel notched with different sizes. Optical images show high stretchability of (j) MFP hydrogel and (k) the notched MFP hydrogel.

the nature of irregular polymerization, the broadly distributed network structure was presented in the CCP hydrogel (Supplementary Fig. 5a). The other control sample of the physically incorporated magnetic PAM hydrogel, denoted as PMP hydrogel, was fabricated by using $\mathrm{N}, \mathrm{N}^{\prime}$-methylene-bis-acrylamide (MBAA) rather than BACA via a similar method to the MFP hydrogel. Herein, the $\mathrm{Fe}_{3} \mathrm{O}_{4} @ \mathrm{Au}$ nanocomposites were randomly aggregated in the PMP hydrogel network arising from no chemical bonds between MBAA and $\mathrm{Fe}_{3} \mathrm{O}_{4} @ \mathrm{Au}$ composites (Supplementary Fig. 5b).

Resulting from chemically crosslinked $\mathrm{Fe}_{3} \mathrm{O}_{4} @ \mathrm{Au}$ composites in the network, the MFP hydrogel exhibited notably mechanical performance. The tensile test quantified that the MFP hydrogel delivered a high tensile stress up to $3.1 \mathrm{MPa}$ at an elongation of $2250 \%$ (Fig. 1h). The MFP hydrogel piece with the length of $2 \mathrm{~cm}$ could be stretched to a length of $>30 \mathrm{~cm}$ without fracture (Fig. 1j). In sharp contrast, the control samples showed much weakened mechanical properties with the tensile stress of $0.5 \mathrm{MPa}$ at the strain of $1100 \%$ for CCP hydrogel and $1.1 \mathrm{MPa}$ at $1300 \%$ for PMP hydrogel (Fig. 1h), indicating the regularly crosslinked structural advantage in MFP hydrogel. Statistics showed much enhanced toughness of $17 \mathrm{MJ} \mathrm{m}^{-3}$ for MFP hydrogel, compared with that of $1.2 \mathrm{MJ} \mathrm{m}^{-3}$ for CCP and $3.5 \mathrm{MJ} \mathrm{m}^{-3}$ for PMP hydrogel (Supplementary Fig. 6). Impressively, the MFP hydrogel demonstrated remarkable notch-insensitivity property, as indicated from high stretchability of the notched gel piece in the optical image (Fig. 1k). As recorded in Fig. 1i, the MFP hydrogels notched with different sizes presented greatly ascended tensile stress when they were stretched until rupture. Even with a large notch of $2 / 3$ of its original width, a large strain of $2050 \%$ was still delivered, corresponding to $91 \%$ of elongation of the original sample. Further comprehensive tests discovered that the MFP hydrogel system with varied content of $\mathrm{Fe}_{3} \mathrm{O}_{4} @ \mathrm{Au}$ composites all behaved tough mechanical performance (Supplementary Fig. 7).

With increasing the content of $\mathrm{Fe}_{3} \mathrm{O}_{4} @ \mathrm{Au}$ nanocomposites in the hydrogel network, the mechanical behavior was improved 
correspondingly. The content of composites was optimized to $2.0 \mathrm{mg} \mathrm{mL}^{-1}$, due to that the excessive nano-crosslinkers were not crosslinked in the polymeric network and weakened the hydrogel strength. Additionally, the MFP hydrogel showed strong compression-resistant capability. As seen from optical images (Supplementary Fig. 8), the highly compressed hydrogel column was rapidly recovered its initial state since the compressive force was released. However, it was observed that the columnar CCP hydrogel was broken into pieces and the PMP hydrogel was fractured when they were under the compression.

Stimuli-triggered healing property of MFP hydrogel. Given that a large number of dynamic Au-SR coordination bonds were homogeneously incorporated in the network, the MFP hydrogel possessed strong healing ability through the reversible deformation/reformation of $\mathrm{Au}-\mathrm{SR}$ bonds under the external stimulus ${ }^{17}$. It was demonstrated that high temperature could trigger the surface reconstruction on the basis of dynamic bonds on/off switching from the surface ${ }^{28}$. However, because of weak thermal conduction of the hydrogel and additional water loss-induced decrease of polymer mobility, little healing was observed when the damaged hydrogel pieces were placed in the oven even for a long time (Supplementary Fig. 9). As illustrated in Fig. 2a, owing to distinct photothermal property of $\mathrm{Au}$ nanoparticles and excellent magneto-thermal property of $\mathrm{Fe}_{3} \mathrm{O}_{4}$ nanospheres from the uniformly dispersed $\mathrm{Fe}_{3} \mathrm{O}_{4} @ \mathrm{Au}$ composites, the MFP hydrogel was expected to be healed inspired by NIR laser or magnetic field. When two individual gel pieces were close in contact and the fractured region was exposed to the NIR laser, it was observed that they were healed in $2 \mathrm{~min}$ and could be stretched to a large strain without any crack (Fig. 2b). Temperature tests by an infrared thermal imager in the inserted images in Fig. $2 \mathrm{C}$ showed that the temperature was up to $43^{\circ} \mathrm{C}$ under the NIR irradiation within $1 \mathrm{~min}$. Reasonably, by improving the content of $\mathrm{Fe}_{3} \mathrm{O}_{4} @ \mathrm{Au}$ composites in the hydrogels, more obvious increase in temperature was detected (Supplementary Fig. 10). Tensile stress-strain curves in Fig. 2c quantified a stretch of $1900 \%$ was maintained for the healed hydrogel, achieving a high healing efficiency of $86.3 \%$ calculated from the strain ratios between the healed and original samples. Furthermore, it was found that the healing efficiency of MFP hydrogel was dependent on the content of the incorporated $\mathrm{Fe}_{3} \mathrm{O}_{4} @ \mathrm{Au}$ composites. As counted in Fig. 2d, when increasing the composite content from 0.4 to $4.0 \mathrm{mg} \mathrm{mL}^{-1}$, the extensibility of the healed hydrogel was $62.5 \%, 69.6 \%, 86.3 \%$, and $81.8 \%$ of the initial length, respectively, owing to the increased density of $\mathrm{Au}-\mathrm{SR}$ sites in the hydrogels. This rapid and high-efficient healing ability outperformed the previously reported self-healing hydrogels with $52-99 \%$ of healing efficiency in $24 \mathrm{~h}^{29-31}$. Furthermore, a MFP hydrogel piece and a CCP hydrogel piece were able to be healed together under NIR laser and behaved strong resistant to stretching deformation (Supplementary Fig. 11), fully confirming the dynamic nature and effectiveness of Au-SR bonds as healing motifs.

In addition to fast and efficient optical healability, the MFP hydrogel demonstrated remotely controllable magnetic healing performance arising from the surface reconstruction triggered by the heat conduction from $\mathrm{Fe}_{3} \mathrm{O}_{4}$ to $\mathrm{Au}$ nanoparticles. Notably, the nanoparticle-composed structure of the $\mathrm{Fe}_{3} \mathrm{O}_{4}$ nanospheres led to the enhanced magneto-thermal effect and activated the kinetics of $\mathrm{Au}-\mathrm{SR}$ bonds. It was detected that the temperature change of the prepared $\mathrm{Fe}_{3} \mathrm{O}_{4}$ nanospheres was increased by $53^{\circ} \mathrm{C}$ within 5 min when placed into the helical coil of the alternating magnetic field generator (Supplementary Fig. 12). When the $\mathrm{Fe}_{3} \mathrm{O}_{4} @ \mathrm{Au}$ composites were crosslinked into the gel network, the MFP hydrogel remained excellent magneto-thermal performance. As shown in Fig. 2e, the temperature of the hydrogels delivered a time-dependent increasing characteristic and with improving the content of the magnetic composites, the real-time temperature increasing was more obvious. Even though $0.3 \mathrm{wt} \%$ of $\mathrm{Fe}_{3} \mathrm{O}_{4} @ \mathrm{Au}$ was contained in the hydrogel, the temperature of the hydrogel column with a diameter of $5 \mathrm{~cm}$ was increased to $45^{\circ} \mathrm{C}$ in $8 \mathrm{~min}$ as revealed by infrared thermal imaging system (Fig. 2f). As exhibited in Fig. 2g, when placing a columnar hydrogel with a large incision in the alternating magnetic field, the hydrogel was healed and could resist the external stretching force. The optical images in Fig. $2 \mathrm{~g}$ clearly showed the fused structure of the damaged interface triggered by magnetic stimulus. To the best of our knowledge, this is the first demonstration of intrinsically magneto-thermal induced healing performance rather than magnetically driven physical contact.

Assembly of omni-healable supercapacitor device. The high mechanical performance and excellent healability promised the MFPbased hydrogel as potential candidates in the assembly of flexible and healable supercapacitor. Especially, when employed for electrodes, the unique $3 \mathrm{D}$ architectures composed of molecular meshes around by polymer chains, interconnected channels and microporous structures offered great advantages on the infiltration of electrolyte and ion transfer in the electrochemical process, in contrast to the conventional electrode configurations (Supplementary Fig. 13a) ${ }^{32}$. To make an active electrode, the conductive polymer, PPy was in situ grown into the MFP framework through soaking the partly-dehydrated MFP hydrogel into the pyrrole solution and the subsequent polymerization initiated by $\mathrm{Fe}^{3+}$. SEM images showed that the MFP-PPy hydrogel maintained the interconnected network structure and a layer of PPy nanoparticles with the uniform size of $100 \mathrm{~nm}$ was coated on the wall of the compartment (Fig. 3b, c). Notably, after the PPy incorporation, the elegant porous structure was still presented in the composites. Compared with the original MFP hydrogel, the pore size of the MFPPPy hydrogel was increased to $10 \mu \mathrm{m}$ arising from the swollen state during the polymerization process. The MFP-PPy hydrogel exhibited high mechanical performance by delivering an elongation of $1650 \%$ (Fig. 3g). These structural advantages guaranteed the MFP-PPy hydrogel electrode fast transports of electron and electrolyte. As illustrated in Fig. 3a, to assemble a healable supercapacitor device, two pieces of MFP-PPy hydrogel electrodes were sandwiched by a MFP hydrogel electrolyte instead of the common PVA gel and AgNW films with the length of $60-100 \mu \mathrm{m}$ and thickness of $1.5-2 \mu \mathrm{m}$ were spray-coated on the electrodes to serve as current collectors (Supplementary Fig. 14a, b). Under the alternating magnetic field, the current collectors, electrodes, and electrolyte could be chemically soldered together by dynamic Ag, Au-SR crosslinkings owing to the magneto-thermal effect. Such an effective interface reconstruction resulted in an integrated device configuration as confirmed by the SEM images (Fig. 3d-f). Furthermore, the structural stability of the current collector embedded in the polymer network of the electrode had been investigated under a continuous stretching from $200 \%$ to $1000 \%$ strain. As shown from SEM images (Supplementary Fig. $14 c-f$ ), with increasing the strains up to a high value of $1000 \%$, the AgNW network was straightened and no visible cracks were observed, indicating the excellent stretchability of the AgNW films as current collectors. More impressively, the mechanical test in Fig. $3 g$ quantified that the assembled supercapacitor delivered a high break elongation of $1200 \%$, indicating its superior stretchability among the reported flexible/stretchable supercapacitors $7,8,33-35$. The optical images in Fig. 3h revealed the good electrical conductivity of the assembled supercapacitor device under multiple deformations including bending, twisting, and stretching with large strains, demonstrating the stable network structure in the MFP-based 

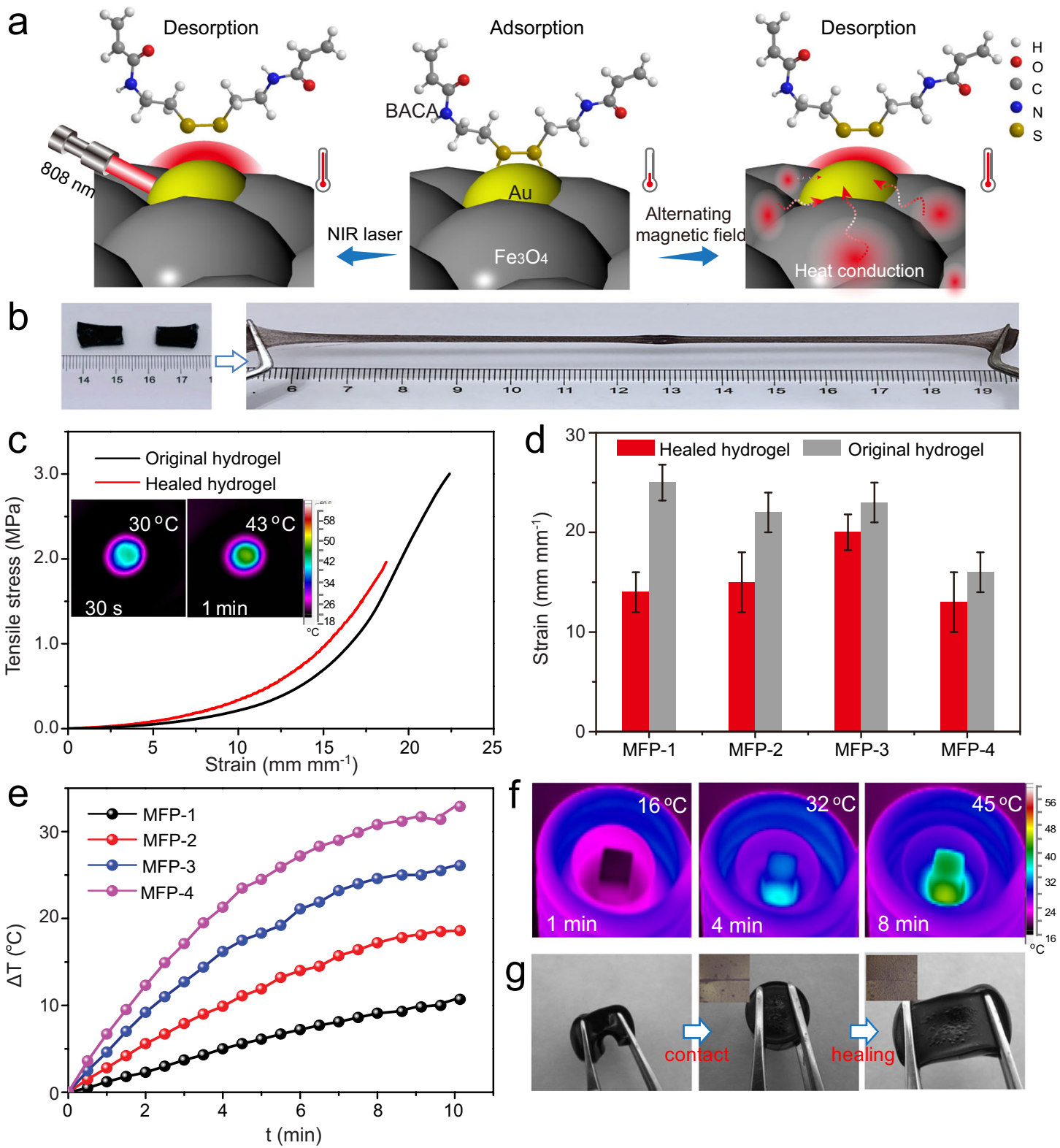

Fig. 2 Stimuli-triggered healing property of MFP hydrogel. a Schematic illustrations of photothermal and magneto-thermal induced self-healing mechanisms. b-d NIR laser-induced self-healing property: b Optical images show high stretchability of the healed hydrogel piece. c Tensile stress-strain curves of original and healed hydrogels. The inserted images show time-dependent temperature changes of hydrogel. $\mathbf{d}$ Strains of original and healed hydrogels with different contents of $\mathrm{Fe}_{3} \mathrm{O}_{4} @$ Au composites. Error bars show the SD with sample size of 3. e-g Magnetically induced self-healing property: e Time-dependent temperature changes of the cylindrical hydrogels with different contents of $\mathrm{Fe}_{3} \mathrm{O}_{4} @ \mathrm{Au}$ composites under alternating magnetic field. f Infrared thermal images of the cylindrical hydrogel. $\mathbf{g}$ Optical images show stretchability of the healed hydrogel. The inserted optical images show the glued structure of the fractured interface.

hydrogels and the integrated device configuration. Based on above results, this work made a good demonstration on the magnetothermal induced assembling of an intrinsically omni-healable supercapacitor based on synergistically controlling constituent, structure, and interfacial interaction of the electrodes, electrolyte, and current collectors.

Multi-responsive healing performance of the supercapacitor. Considering the intrinsically healable hydrogels employed as electrodes and electrolyte, the assembled supercapacitor showed great self-healing capability when got damaged (Fig. 4a). Typically, combined with the thermal-triggered reversible and dynamic feature of metal-thiolate interaction, the AgNWs, $\mathrm{Au}$ nanoparticles, $\mathrm{Fe}_{3} \mathrm{O}_{4}$ nanospheres, and PPy nanoparticles inside the device performed as thermal sources would facilitate the interfacial fusion of the damaged parts under the external stimuli. During the heating process, the mobility of sulfur-atom-ended polymer network was greatly improved, which enabled the reconstruction of polymer chains on the metal surface from the neighboring parts for secondary combination. As schematically illustrated in Fig. 4b, the crack interfaces among the current collector/electrode/electrolyte layers of the supercapacitor can be healed owing to the interfacial reconstruction of dynamic $\mathrm{Au}$, Ag-SR bonds inspired by distinct photothermal, galvano-thermal, and magneto-thermal effects under NIR laser irradiation, electric current, and alternating magnetic field, respectively. 

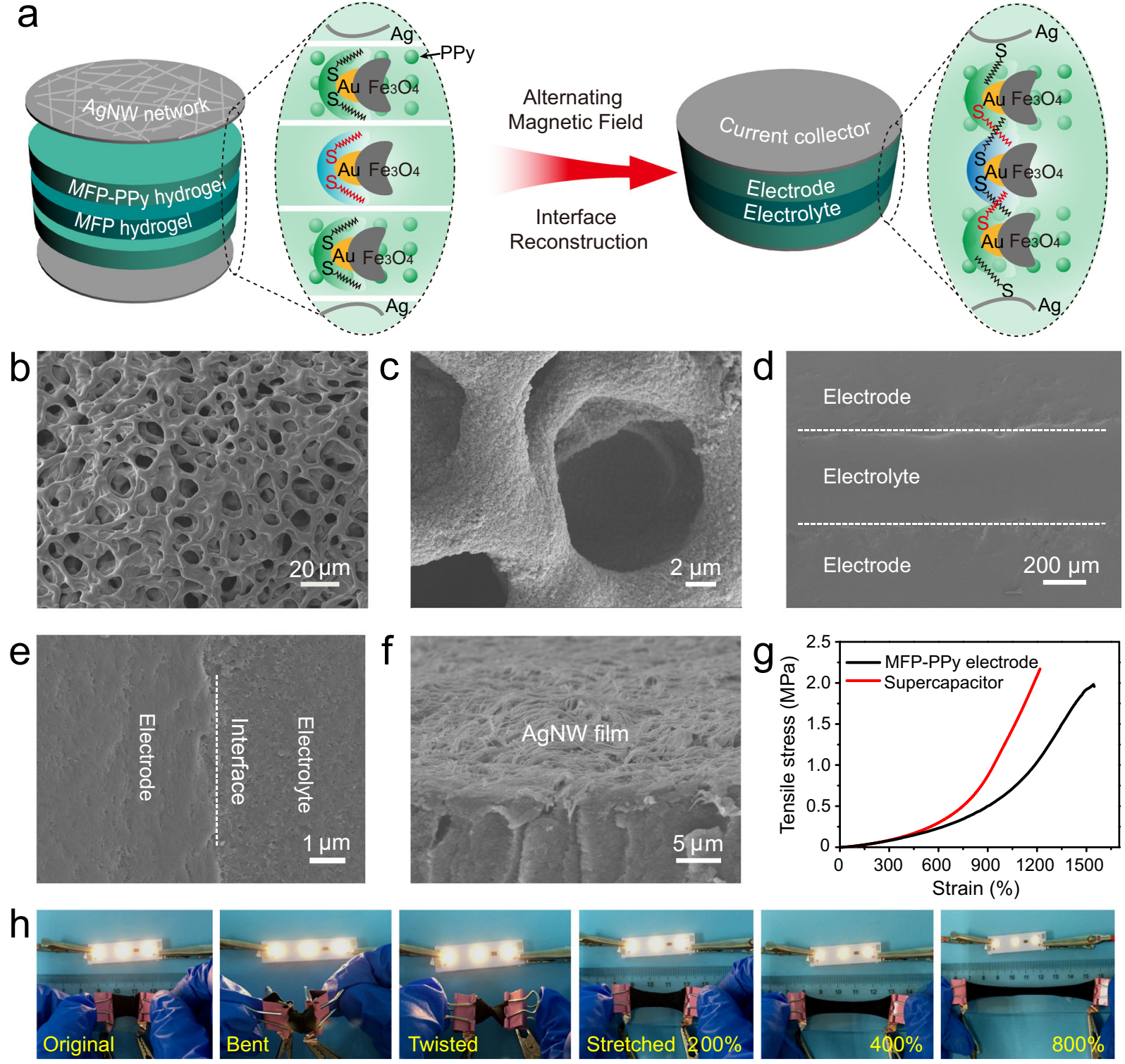

Fig. 3 Assembly of the healable supercapacitor device. a Schematic illustrations of the magneto-thermal induced assembly of the supercapacitor by sandwiching two MFP-PPy hydrogel electrodes with a MFP hydrogel electrolyte and spraying AgNW film layers as current collectors. The device presents an integrated configuration owing to the metal-thiolate bond-triggered interface reconstruction under alternating magnetic field. SEM image (b) and enlarged SEM image (c) of MFP-PPy hydrogel electrode. d Cross-sectional SEM image of the supercapacitor. e Enlarged SEM image of the electrode/ electrolyte interface of the supercapacitor. $\mathbf{f}$ SEM image of the AgNW film coated on the electrode. $\mathbf{g}$ Tensile stress-strain curves of the MFP-PPy hydrogel electrode and the assembled supercapacitor. $\mathbf{h}$ Optical images show good electrical conductivity of the supercapacitor at multiple deformations including bending, twisting, and stretching with high strains.

The capacitive performance of the assembled supercapacitor device was evaluated by cyclic voltammetry (CV) and galvanostatic charge-discharge (GCD) measurements using a two-electrode method. As shown in Fig. 5a, the quasi-rectangular CV profiles with the mirror-image symmetry were presented at low scan rates from 10 to $50 \mathrm{mV} \mathrm{s}^{-1}$. At a higher scan rate of $100 \mathrm{mV} \mathrm{s}^{-1}$, the diffusion limitation and the increasing transfer resistance resulted in the CV curve deviating from the rectangular shape. The surrounded CV areas dependent on the scan rates suggested ideal capacitive behaviors of the supercapacitor. Figure $5 \mathrm{~b}$ showed the GCD curves with typical symmetric triangular shapes at the current densities from 3 to $50 \mathrm{~mA} \mathrm{~cm}^{-2}$ and a potential window of $0-0.8 \mathrm{~V}$. It was calculated that the areal capacitance reached $1264 \mathrm{mF} \mathrm{cm}^{-2}$ at a current density of $3 \mathrm{~mA} \mathrm{~cm}^{-2}$, which was the highest value among the previously reported healable supercapacitors (Supplementary Table 1). Notably, the supercapacitor demonstrated excellent rate performance on areal capacitance (Fig. $5 \mathrm{c}$ and Supplementary Fig. 15). When increasing the current densities from 10 to $50 \mathrm{~mA} \mathrm{~cm}^{-2}$, the areal capacitances were stable at each rate. Even at a high current density of $50 \mathrm{~mA} \mathrm{~cm}^{-2}$, a capacitance of $309 \mathrm{mF} \mathrm{cm}^{-2}$ was estimated, which was greater than that of the previously reported

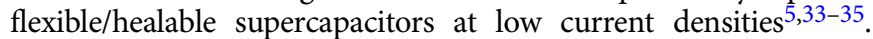
Since the current density was back to $10 \mathrm{~mA} \mathrm{~cm}^{-2}$, its capacitance was recovered to $727 \mathrm{mF} \mathrm{cm}^{-2}$. In the extended GCD tests at a current density of $10 \mathrm{~mA} \mathrm{~cm}-2$, the device showed capacitance retention of $96.1 \%$ after 5000 charge-discharge cycles (Supplementary Fig. 16a). However, the high proportion of interconnected porous structure and moderate density of PPy enabled the assembled supercapacitor with a limited volumetric capacitance 


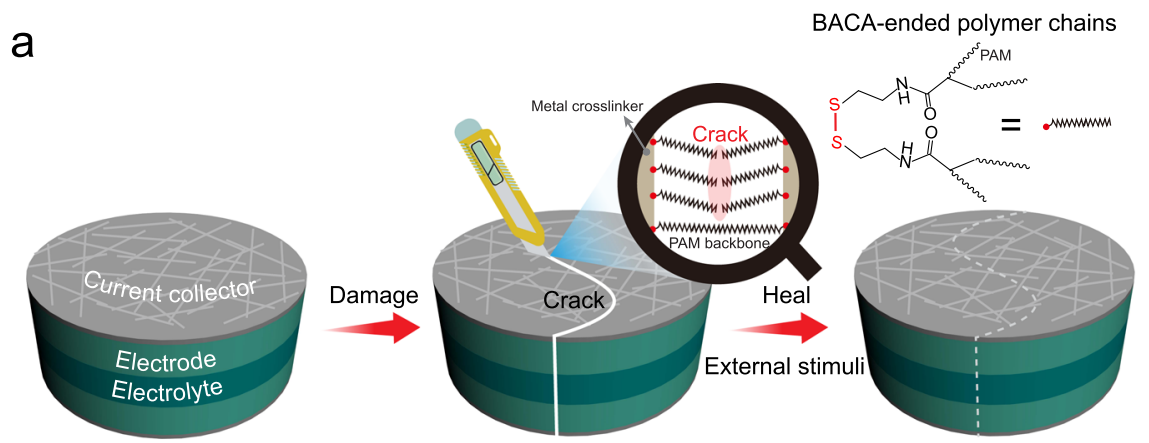

External stimuli: NIR laser/Alternating magnetic field/Electric current.

b

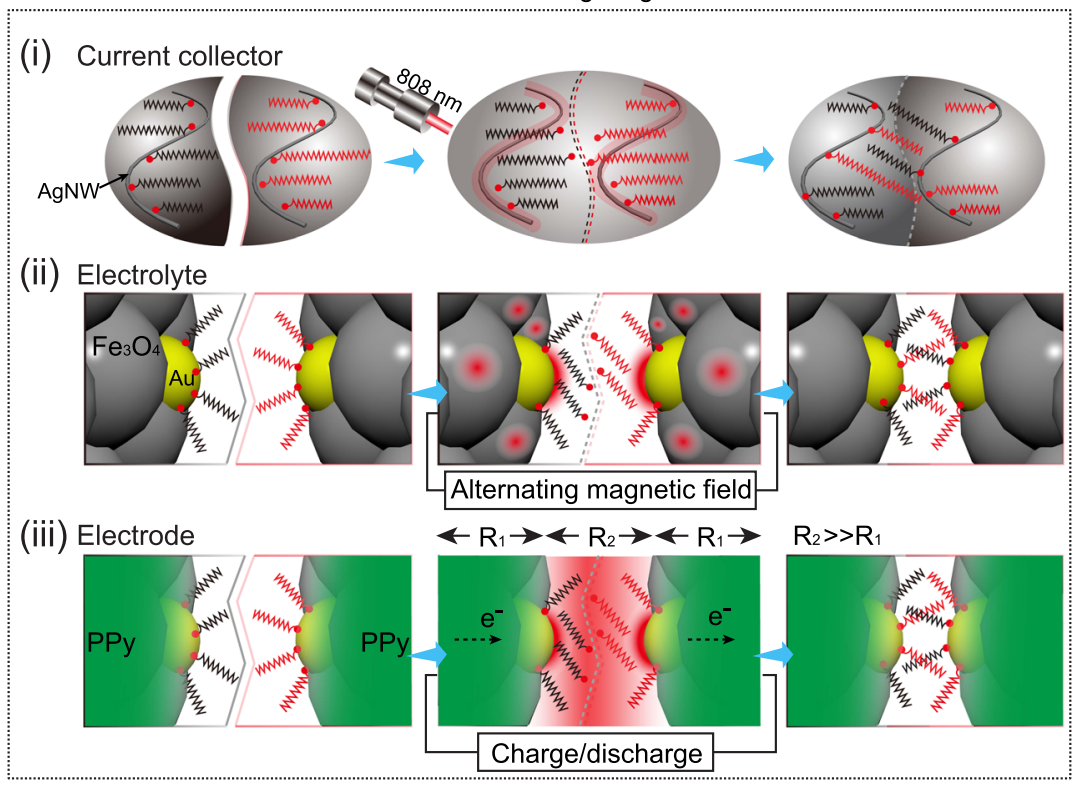

Dynamic Ag, Au-SR bond $\longrightarrow$ Interface reconstruction

Fig. 4 Schematic illustrations of self-healing mechanism of supercapacitor. a Scheme of the self-healing process. b Schematic illustrations for the healing mechanism of the damaged supercapacitor under the external stimuli, taking optical healing of current collector, magnetic healing of electrolyte, and electrical healing of electrode as examples. The dynamic $\mathrm{Ag}, \mathrm{Au}-\mathrm{SR}$ bond-induced interface reconstruction results in the healing of the crack region under the photothermal, magneto-thermal, and galvano-thermal effects.

(22.3 $\mathrm{F} \mathrm{cm}^{-3}$ at $10 \mathrm{~mA} \mathrm{~cm}^{-2}$ ) (Fig. $5 \mathrm{c}$ ). To reveal the anti-drying stability of the hydrogel-based supercapacitor, the electrochemical performance of the assembled device during the drying process was systematically investigated. Firstly, the influence of dehydration of hydrogel electrolyte on the capacitance performance was studied. By real-time monitoring the water variation of the electrolyte exposed to the air at room temperature with the humidity of $40-50 \%$, a relatively slow dehydration was indicated with $\sim 25 \%$ of water loss in $86 \mathrm{~h}$ (Supplementary Fig. 17a). The capacitive performance of the assembled supercapacitor was gradually decreased with $71 \%$ of original capacitance retained correspondingly (Supplementary Fig. 17b). Benefited from the densely crosslinked network with enhanced capability of water retention, an integrated supercapacitor when exposed to the air also exhibited a slow dehydration behavior (Supplementary Fig. 18a). When the water content was decreased from the original $74 \%$ to $51 \%$ in $72 \mathrm{~h}, 64 \%$ of the specific capacitance was still retained (Supplementary Fig. 18b). In contrast, the two comparative supercapacitors assembled from the CCP/PPy and PMP/PPy electrodes delivered much weakened capacitive performances as indicated from the irregular rectangular CV shapes with the smaller areas (Supplementary Fig. 19a). Concretely, calculated from the corresponding GCD curves, the areal capacitances of 242 and $422 \mathrm{mF} \mathrm{cm}^{-2}$ were determined for the CCP/PPy and PMP/
PPy supercapacitors, respectively (Supplementary Fig. 19b), much lower than $1264 \mathrm{mF} \mathrm{cm}^{-2}$ of the MFP-PPy device. As revealed in the electrochemical impedance spectroscopy (EIS) spectra (Supplementary Fig. 20), the MFP-PPy supercapacitor presented excellent electrical conductivity by delivering a low chargetransfer resistance of $0.12 \Omega$ as estimated from the semicircle diameter in the high-frequency region. Furthermore, the straight line in a steep slope in the low-frequency region indicated a small electrolyte diffusion resistance and good capacitive behavior. Contrarily, the two reference supercapacitors exhibited much larger resistance, indicating inferior electrical conductivities (Supplementary Fig. 20). In contrast to the CCP/PPy and PMP/ PPy, the employment of BACA-modified $\mathrm{Fe}_{3} \mathrm{O}_{4} @ \mathrm{Au}$ nanocomposites as crosslinkers offered the advantage of uniform and controlled porous morphology in the MFP hydrogel. The detailed information on the internal porous morphology was investigated through mercury intrusion porosimeter (MIP) (Supplementary Fig. 13b). Compared to the CCP/PPy and PMP/PPy electrodes, the MFP-PPy hydrogel electrode presented more uniform pore structures, as indicated from the narrower pore distributions in different regions of the MIP analysis, which was in favor of electron transport and electrolyte diffusion during the electrochemical process. Based on these electrochemical analyses, the combined merits of high electrical conductivity and an integrated 

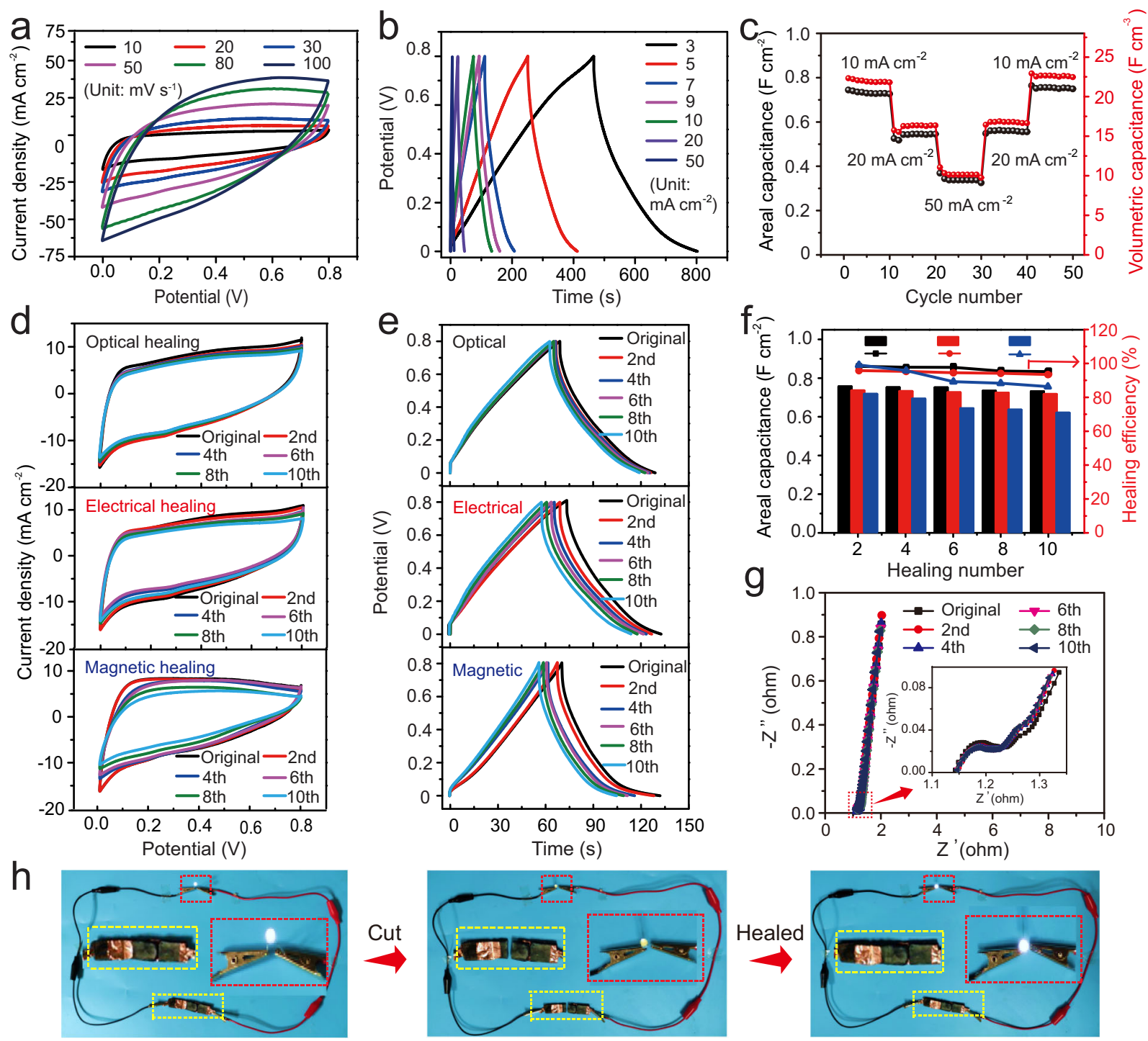

Fig. 5 Multi-responsive healing performance of the supercapacitor. a CV curves at different scan rates. b GCD curves at different current densities c Rate performance of areal and volumetric capacitances at current density ranging from 10 to $50 \mathrm{~mA} \mathrm{~cm}^{-2}$. Optical, electrical, and magnetic healing process over 10 cutting-healing cycles: $\mathbf{d}$ CV curves at a scan rate of $10 \mathrm{mV} \mathrm{s}^{-1}, \mathbf{e ~ G C D ~ c u r v e s ~ a t ~ a ~ c u r r e n t ~ d e n s i t y ~ o f ~} 10 \mathrm{~mA} \mathrm{~cm}{ }^{-2}$, $\mathbf{f}$ areal capacitance and healing efficiency during optical (black), electrical (red), and magnetic (blue) healing process, and $\mathbf{g}$ ElS spectra during the optical healing processes. The inset showing the enlarged view of the rectangular area in the high-frequency region. $\mathbf{h}$ Optical images show the lighting of a LED bulb by three supercapacitors connected in series during the cutting-healing process. The yellow and red squares are the enlarged photographs of the capacitor and bulb, respectively.

device configuration along with the structural advantages of the electrode and electrolyte resulted in superior performance of the MFP-PPy supercapacitor.

Further electrochemical measurements were carried out to demonstrate multi-responsive healing performance of the MFPPPy assembled supercapacitor under the optical, electrical, and magnetic stimuli, respectively. As shown in Fig. 5d, the device presented the nearly overlapped regular-shaped $\mathrm{CV}$ curves before and after different cutting-healing cycles at a scan rate of $10 \mathrm{mV} \mathrm{s}^{-1}$, indicating its non-deteriorated capacitive performances under three external stimuli, respectively. Moreover, the GCD curves of the device were highly consistent in both shape and discharging time before and after healing cycles at a current density of $10 \mathrm{~mA} \mathrm{~cm}^{-2}$, suggesting no obvious degradation in capacitance (Fig. 5e). In comparison with magnetic and electric healing, the optically healed device presented the lowest deviation on the GCD curves arising from the highest healing efficiency of NIR laser irradiation with the strongest heating capability (Supplementary Fig 21a). In addition,
$86.3 \%$ of mechanical healing efficiency in 2 min proved the highly efficient healing performance under the stimulus of NIR (Supplementary Fig. 21b). For clearness, Fig. 5f showed the areal capacitances and healing efficiencies of the device during the cutting-healing cycles under different stimuli calculated from the GCD curves. After the 10th optical, electrical and magnetic healing processes, the supercapacitor restored the areal capacitance of 710 , 702 , and $609 \mathrm{mF} \mathrm{cm}^{-2}$ at the current density of $10 \mathrm{~mA} \mathrm{~cm}^{-2}$, which was estimated to be $95.2 \%, 93.8 \%$, and $86.1 \%$ in healing efficiency, respectively. Compared with NIR laser-induced healing, the smaller capacitance retention was demonstrated during the magnetic healing process, which was resulted from moderate magnetic heating capability under the mild magnetic field, as confirmed by $52 \%$ of mechanical healing efficiency in $10 \mathrm{~min}$ (Supplementary Fig. 21b). Considering the healing mechanism of heating-induced metalcoordination interface reconstruction, the heating capability of the Au surface in the gel determined the healing efficiency. For the optical healing process, the heating was directly produced from the 
Au nanoparticles with the notable photothermal property, resulting in a rapid and highly efficient healing. In the magnetic healing process, the heating of the $\mathrm{Au}$ surface was indirect, which was transferred from the neighboring $\mathrm{Fe}_{3} \mathrm{O}_{4}$ spheres. Moreover, unsatisfactory thermal-conduction of $\mathrm{Fe}_{3} \mathrm{O}_{4}$ led to heat loss inevitably. Herein, a bit deteriorated electrical healing efficiency was because of unavoidable thermal loss in the heat transfer between PPy and Au nanoparticles, and continuous swelling and shrinking of the polymer during the charge-discharge process for the electrical healing 36 .

EIS spectra of the device after different healing cycles coincided with the original profile, suggesting good restorations of the electrical conductivity during the stimulus-inspired healing process (Fig. 5g and Supplementary Fig. 22). The small impedance arcs in the high-frequency region and small intercept along $\mathrm{Z}^{\prime}$ indicated low charge-transfer resistance between the electrolyte and electrode and equivalent series resistance of the healed devices. The nearly vertical profile for the healed supercapacitors in the low-frequency region revealed an ideal capacitive behavior. SEM images showed that the broken interface of the device was glued together and presented a welldefined interconnected network, confirming its effective healing behavior (Supplementary Fig. 23). The optical images exhibited the stable brightness of the light-emitting diode (LED) bulbs even when the healed device was stretched at high strains (Supplementary Fig. 24), revealing a highly efficient healing of the MFPPPy supercapacitor. Furthermore, the healed supercapacitor showed excellent long-term cycling stability, as proved by 93.4\% of capacitance retention after 5000 GCD cycles at a current density of $10 \mathrm{~mA} \mathrm{~cm}^{-2}$ (Supplementary Fig. 16b). Consequently, the supercapacitors could power the LED bulb after the cutting-healing operation (Fig. 5h).

\section{Discussion}

As compared with previously reported self-healable supercapacitors, several advantages can be easily found and summarized. First, the healable layers including both electrodes and electrolyte were combined through the healing-induced strategy under external stimuli for all-solid-state flexible and stretchable supercapacitors ${ }^{37}$. This integrated configuration containing reversible interfaces with rich metal-thiolate interaction between current collectors, hydrogel electrodes, and electrolyte layer, enables the device with excellent capacitance performance as well as great stretchability and healing behaviors. Especially, the exclusive interfacial interaction of metal-thiolate coordination throughout the devices facilitates the healing performance in a rapid and highly efficient way within single stimulus (Supplementary Table 1). Second, the fabricated supercapacitors show great promise as power sources in case of being integrated with other active devices for realization of wearable electronics since their professional flexibility and stretchability ${ }^{38,39}$. In contrast to other healable supercapacitors with single-stimulus responsive property reported, the multi-stimulus induced self-healing performance of our fabricated supercapacitors would provide additional advantages when the neighboring active devices are also stimulus-responsive, for example, sensors. Depending on the components of the active devices, appropriate stimulus can be chosen and performed to heal the damaged region, accompanied with little triggering effect on the neighboring devices. As such, the fabricated supercapacitors have been demonstrated to be selfhealable under various external stimuli including NIR light, electricity, and alternating magnetic field. Based on the device integration, the healing experiments can be carried out independently, far away from the active device. It is noteworthy that the stimuli of light and magnet can be performed in a remote and located way, which can further decrease the undesirable responsiveness. Last but not the least, these synthetic materials with professional mechanical and self-healing performances would present great significance to the sustainable development and environmental protection. Despite higher cost than the biomassbased counterparts ${ }^{40-42}$, the synthetic materials could provide great advantages on the structural design and interfacial control for the purpose of professional mechanical and functional properties, such as mechanical strength, stretchability, and selfhealing behavior. Specially, the excellent stretchable performance enables the integrated device with broad applications in versatile environments under multiple mechanical deformations. The realization of materials with self-healing capability, as an efficient and cost-effective alternative, can increase the lifetime of the structures and decrease their maintenance cost. Such an intrinsically healable design guaranteed the integrated structural configuration and good recovery in electrical conductivity and mechanical property, and therefore promised high capacitive performance and healing efficiency of the device, confirming the MFP hydrogel-assembled supercapacitor as one of the best performers among the flexible and healable supercapacitors.

In conclusion, we have demonstrated an optically, electrically, and magnetically responsive self-healing supercapacitor device with the integrated configuration soldered by dynamic Ag, Au-SR bonds assembled from the MFP-based hydrogel electrodes and electrolyte and AgNW films as current collectors. Resulting from the chemically crosslinked $\mathrm{Fe}_{3} \mathrm{O}_{4} @ \mathrm{Au}$ nanocomposite in the polymer network, the MFP hydrogel exhibited tough mechanical strength with a high strechability of $2250 \%$ and notable notchinsensitivity. Furthermore, its notable photothermal and magneto-thermal properties enabled the hydrogel with fast optical and remotely controllable magnetic healing relied on high-density dynamic Au-SR bonds. With the merits of the porous structure, strong mechanical property and intrinsic healability of the electrode and electrolyte, the assembled supercapacitor delivered a largest areal capacitance of $1264 \mathrm{mF} \mathrm{cm}^{-2}$ among the reported healable supercapacitors and superhigh device-level stretchability of $1200 \%$. As a demonstration of triplyresponsive self-healing supercapacitor, the device presented excellent healing performance with $\sim 90 \%$ of capacitances restored over ten optical, electrical, and magnetic healing cycles, respectively. This intrinsic self-healing integration strategy along with the advanced electrode and electrolyte proposed here allows for assembling multifunctional and high-performance supercapacitor devices under precise interfacial controls, promising great potentials in powering next-generation wearable and portable electronic devices.

\section{Methods}

Preparation of $\mathbf{F e}_{\mathbf{3}} \mathbf{O}_{\mathbf{4}} @ \mathbf{A u}$ nanocomposites. In a typical synthesis, $\mathrm{Fe}_{3} \mathrm{O}_{4}$ nanospheres were firstly synthesized by a solvothermal method. In all, $1.8 \mathrm{~g}$ of $\mathrm{FeCl}_{3} \cdot 6 \mathrm{H}_{2} \mathrm{O}$ was dissolved in $40 \mathrm{~mL}$ of ethylene glycol at room temperature to form a clear solution. Then, $0.27 \mathrm{~g}$ of trisodium citrate, $1 \mathrm{~g}$ of polyvinyl pyrrolidone, and $2.4 \mathrm{~g}$ of sodium acetate were successively added into above solution. After stirring for $2 \mathrm{~h}$, the obtained mixture was transferred to a sealed Teflon-lined stainless-steel autoclave with a capacity of $50 \mathrm{~mL}$ and heated at $200^{\circ} \mathrm{C}$ for $10 \mathrm{~h}$. After cooling to room temperature, the products were washed by water and ethanol three times with the assistance of a magnet. In the following, in situ reduction of Au nanoparticles onto $\mathrm{Fe}_{3} \mathrm{O}_{4}$ nanospheres was carried out. Typically, $2 \mathrm{mg} \mathrm{mL} \mathrm{m}^{-1}$ of $\mathrm{Fe}_{3} \mathrm{O}_{4}$ nanospheres were dispersed into the solution of ethanol and ammonia under ultrasonication. Then, $300 \mu \mathrm{L}$ of $\mathrm{HAuCl}_{4}(1 \mathrm{wt} \%)$ was added into the above dispersion quickly. With stirring for $2 \mathrm{~h}$, the $\mathrm{Fe}_{3} \mathrm{O}_{4} @ \mathrm{Au}$ composites were collected by a magnet, cleaned with water and ethanol three times and dried under vacuum at $60^{\circ} \mathrm{C}$ for use

Preparation of MFP hydrogel. In a typical procedure, the as-prepared $\mathrm{Fe}_{3} \mathrm{O}_{4} @ \mathrm{Au}$ nanocomposites were homogeneously dispersed in $5 \mathrm{~mL}$ of aqueous solution. Then, the desired amount of BACA was added to the above solution to obtain the BACAmodified $\mathrm{Fe}_{3} \mathrm{O}_{4} @ \mathrm{Au}$ composites as the multifunctional crosslinkers for the 
subsequent polymerization. Next, $1.0 \mathrm{~g}$ of AAm, $0.02 \mathrm{~g}$ of KPS, and $20 \mu \mathrm{L}$ of TEMED were added with ultrasonication until all reagents were dissolved. Then, $\mathrm{N}_{2}$ bubbling was conducted to remove the dissolved $\mathrm{O}_{2}$. After that, the mixture was degassed in a vacuum drying oven. With the polymerization at room temperature for $24 \mathrm{~h}$, the magnetic composite hydrogel was obtained. Similarly, several magnetic hydrogels were fabricated by using different contents of $\mathrm{Fe}_{3} \mathrm{O}_{4} @ \mathrm{Au}$ composites, that was $0.4,1.0,2.0$, and $4.0 \mathrm{mg} \mathrm{mL}^{-1}$, denoted as MFP- $n$ $(n=1-4)$. Furthermore, the reference sample, physically-incorporated magnetic PAM hydrogel was also prepared by using the organic crosslinker, MBAA, instead of BACA, denoted as PMP hydrogel. In this case, $\mathrm{Fe}_{3} \mathrm{O}_{4} @ \mathrm{Au}$ composites were not chemically crosslinked in the network because of no chemical interactions between MBAA and $\mathrm{Fe}_{3} \mathrm{O}_{4} @ \mathrm{Au}$ composites. The conventional chemically crosslinked polymeric hydrogel was also prepared as the other control sample by using BACA as chemical crosslinker without the addition of $\mathrm{Fe}_{3} \mathrm{O}_{4} @ \mathrm{Au}$ composites, denoted as CCP hydrogel.

Preparation of MFP-PPy hydrogel electrode. The MFP hydrogel was firstly dehydrated at room temperature for $24 \mathrm{~h}$ to remove part of the water from the gel. Then, the hydrogel piece was soaked in $0.5 \mathrm{~mol} \mathrm{~L}^{-1}$ of pyrrole solution for $6 \mathrm{~h}$ to achieve the swelling equilibrium. The swollen hydrogel was placed into the yellow $\mathrm{FeCl}_{3}$ solution $\left(1.0 \mathrm{~mol} \mathrm{~L}^{-1}\right)$. After the polymerization at room temperature for $12 \mathrm{~h}$, the MFP-PPy hydrogel electrode was prepared with washing to remove the impurities on the surface. The mass loading and corresponding mass fraction of the PPy on the MFP hydrogel were $18.2 \pm 3 \mathrm{mg} \mathrm{cm}^{-3}$ and $\sim 5 \pm 1 \%$ (dry sample), respectively.

Healing property measurements. The optical-induced healing process was executed using a NIR laser (MDL-III-808) with the wavelength of $808 \mathrm{~nm}$ and the power of $1.5 \mathrm{~W}$. The hydrogels were cut into two pieces by a sharp blade. With the separated parts put into contact, the laser was exposed on the joint of hydrogels at a distance of $10 \mathrm{~cm}$ with the spot area of $1 \mathrm{~cm} \times 0.5 \mathrm{~cm}$ at room temperature. The magnetic triggered healing process was carried out on a gel cylinder with a radius incision by a sharp blade. The fractured sample was healed when placed into the helical coil of a home-made alternating magnetic field generator at $315 \mathrm{KHz}$, $25 \mathrm{kA} \mathrm{m}^{-1}$. The exact healing time including the heating and cooling time for the MFP hydrogel-based supercapacitors was $2 \mathrm{~min}, 6 \mathrm{~min}$, and $10 \mathrm{~min}$ in the external stimuli of NIR light, electric current, and alternating magnetic field, respectively.

\section{Assembly and electrochemical measurements of healable supercapacitor} device. The supercapacitor device was assembled by sandwiching two pieces of MFP-PPy hydrogel electrodes ( $1 \mathrm{~mm}$ thick) with MFP hydrogel electrolyte $(1 \mathrm{~mm}$ thick) and spray-coating AgNW films on the electrodes as current collectors. Placing at a helical coil of the alternating magnetic field, the layers of current collector, electrode and electrolyte were chemically soldered together. Prior to device assembly, the electrode and electrolyte hydrogels were soaked in $1.0 \mathrm{~mol} \mathrm{~L}^{-1}$ of $\mathrm{Na}_{2} \mathrm{SO}_{4}$ aqueous solution for $2 \mathrm{~h}$. To improve the capacitive performance, the thickness of the device was reduced to be $1 \mathrm{~mm}$ by the compression between two metal plates. All the electrochemical tests including CV, GCD curves and EIS spectra were performed using two-electrode system on a $\mathrm{CHI} 760 \mathrm{E}$ electrochemical workstation at room temperature. $\mathrm{CV}$ curves were recorded at various scan rates from 10 to $100 \mathrm{mV} \mathrm{s}^{-1}$ and the potential range of $0-0.8 \mathrm{~V}$. GCD tests were carried out at the current densities from 3 to $50 \mathrm{~mA} \mathrm{~cm}^{-2}$ with the potential range of $0-0.8 \mathrm{~V}$. EIS spectra were tested in the frequency range of $10 \mathrm{mHz}-100 \mathrm{kHz}$. The areal capacitance was calculated based on the equation:

$$
C=\frac{I \times \Delta t}{S \times \Delta U},
$$

where $I$ is the discharge current, $\Delta t$ is the discharge time and $\Delta U$ is the voltage window, and $S$ is the area of the electrode material. The volumetric capacitance was calculated based on the equation:

$$
C=\frac{I \times \Delta t}{V \times \Delta U},
$$

where $V$ is the volume of the electrode material. The optical and magnetic healing of the damaged device was carried out with the help of NIR laser irradiation and alternating magnetic field generator, respectively. The real-time electrical healing of the damaged device was performed through the charge-discharge cycles at a current of $50 \mathrm{~mA}$. For healing cycles, the devices were cut at the same position.

Materials characterization. SEM images were characterized by a field-emission scanning electron microscopy (Zeiss, Merlin Compact) at an acceleration voltage of $5 \mathrm{kV}$. High-resolution TEM images and the elemental mapping profiles were carried out on a JEM-2100F field-emission transmission electron microscopy equipped with an Oxford Inca energy instrument at $200 \mathrm{kV}$. XPS were recorded on an ESCALab MKII X-ray photoelectron spectrometer by means of a monochromatic X-ray source. XRD patterns were recorded on a Philips X'Pert PRO MPD X-ray diffractometer by using $\mathrm{Cu}$ Ka radiation. MIP study was performed on an AutoPore IV 9510 for detailed analysis of the pore morphology of hydrogels. All the mechanical curves were recorded on the Instron 5965A mechanical testing system with a $100 \mathrm{~N}$ load cell. The tensile experiments were conducted on the hydrogel pieces with a length of $15 \mathrm{~mm}$, width of $5 \mathrm{~mm}$, and thickness of $2 \mathrm{~mm}$ at a stretch rate of $10 \mathrm{~mm} \mathrm{~min}^{-1}$. Infrared thermal images were conducted on a Fluke Ti400 infrared imager.

\section{Data availability}

All data generated or analyzed during this study are included in the published article and its Supplementary Information. Raw data are available on reasonable request from the corresponding authors (H.-P.C. or S.-H.Y.).

Received: 7 January 2021; Accepted: 27 May 2021; Published online: 14 July 2021

\section{References}

1. White, S. R. et al. Autonomic healing of polymer composites. Nature 409, 794-797 (2001).

2. Chen, X. et al. A thermally re-mendable cross-linked polymeric material. Science 295, 1698-1702 (2002).

3. Hager, M. D., Greil, P., Leyens, C., van der Zwaag, S. \& Schubert, U. S. Selfhealing materials. Adv. Mater. 22, 5424-5430 (2010).

4. Wang, H. et al. A mechanically and electrically self-healing supercapacitor. Adv. Mater. 26, 3638-3643 (2014).

5. Huang, Y. et al. Magnetic-assisted, self-healable, yarn-based supercapacitor. ACS Nano 9, 6242-6251 (2015).

6. Tao, F., Qin, L., Wang, Z. \& Pan, Q. Self-healable and cold-resistant supercapacitor based on a multifunctional hydrogel electrolyte. ACS Appl. Mater. Interfaces 9, 15541-15548 (2017).

7. Wang, S. et al. Highly stretchable and self-healable supercapacitor with reduced graphene oxide based fiber springs. ACS Nano 11, 2066-2074 (2017)

8. Huang, Y. et al. A self-healable and highly stretchable supercapacitor based on a dual crosslinked polyelectrolyte. Nat. Commun. 6, 10310 (2015).

9. Wang, Z. \& Pan, Q. An omni-healable supercapacitor integrated in dynamically cross-linked polymer networks. Adv. Funct. Mater. 27, 1700690 (2017).

10. Cordier, P., Tournilhac, F., Soulie-Ziakovic, C. \& Leibler, L. Self-healing and thermoreversible rubber from supramolecular assembly. Nature 451, 977-980 (2008).

11. Wei, Z. et al. Self-healing gels based on constitutional dynamic chemistry and their potential applications. Chem. Soc. Rev. 43, 8114-8131 (2014).

12. Kang, J. et al. Tough and water-insensitive self-healing elastomer for robust electronic skin. Adv. Mater. 30, 1706846 (2018).

13. Wu, J., Cai, L.-H. \& Weitz, D. A. Tough self-healing elastomers by molecular enforced integration of covalent and reversible networks. Adv. Mater. 29, 1702616 (2017).

14. Amamoto, Y., Kamada, J., Otsuka, H., Takahara, A. \& Matyjaszewski, K. Repeatable photoinduced self-healing of covalently cross-linked polymers through reshuffling of trithiocarbonate units. Angew. Chem. Int. Ed. 50, 1660-1663 (2011)

15. Mozhdehi, D., Ayala, S., Cromwell, O. R. \& Guan, Z. Self-healing multiphase polymers via dynamic metal-ligand interactions. J. Am. Chem. Soc. 136, 16128-16131 (2014)

16. Li, C.-H. et al. A highly stretchable autonomous self-healing elastomer. Nat. Chem. 8, 619-625 (2016).

17. Qin, H. et al. Dynamic Au-thiolate interaction induced rapid self-healing nanocomposite hydrogels with remarkable mechanical behaviors. Chem 3, 691-705 (2017).

18. Burnworth, M. et al. Optically healable supramolecular polymers. Nature $\mathbf{4 7 2}$ 334-337 (2011).

19. $\mathrm{Hu}, \mathrm{K}$. et al. A novel magnetic hydrogel with aligned magnetic colloidal assemblies showing controllable enhancement of magnetothermal effect in the presence of alternating magnetic field. Adv. Mater. 27, 2507-2514 (2015).

20. Crippa, F. et al. Dynamic and biocompatible thermo-responsive magnetic hydrogels that respond to an alternating magnetic field. J. Magn. Magn. Mater. 427, 212-219 (2017).

21. Li, Y. et al. Magnetic hydrogels and their potential biomedical applications. Adv. Funct. Mater. 23, 660-672 (2013).

22. Ilg, P. Stimuli-responsive hydrogels cross-linked by magnetic nanoparticles. Soft Matter 9, 3465-3468 (2013).

23. Zhao, W., Odelius, K., Edlund, U., Zhao, C. \& Albertsson, A.-C. In situ synthesis of magnetic field-responsive hemicellulose hydrogels for drug delivery. Biomacromolecules 16, 2522-2528 (2015).

24. Lao, L. L. \& Ramanujan, R. V. Magnetic and hydrogel composite materials for hyperthermia applications. J. Mater. Sci. Mater. Med. 15, 1061-1064 (2004).

25. Zhang, Y. L. et al. A magnetic self-healing hydrogel. Chem. Commun. 48, 9305-9307 (2012) 
26. Yu, C., Wang, C.-F. \& Chen, S. Robust self-healing host-guest gels from magnetocaloric radical polymerization. Adv. Funct. Mater. 24, 1235-1242 (2014).

27. Deng, Y., Qi, D., Deng, C., Zhang, X. \& Zhao, D. Superparamagnetic highmagnetization microspheres with an $\mathrm{Fe}_{3} \mathrm{O}_{4} @ \mathrm{SiO}_{2}$ core and perpendicularly aligned mesoporous $\mathrm{SiO}_{2}$ shell for removal of microcystins. J. Am. Chem. Soc. 130, 28-29 (2008)

28. Yin, Y. \& Alivisatos, A. P. Colloidal nanocrystal synthesis and the organic-inorganic interface. Nature 437, 664-670 (2004).

29. Miyamae, K., Nakahata, M., Takashima, Y. \& Harada, A. Self-healing, expansion-contraction, and shape-memory properties of a preorganized supramolecular hydrogel through host-guest interactions. Angew. Chem. Int. Ed. 54, 8984-8987 (2015).

30. Phadke, A. et al. Rapid self-healing hydrogels. Proc. Natl Acad. Sci. USA 109, 4383-4388 (2012).

31. Sun, T. L. et al. Physical hydrogels composed of polyampholytes demonstrate high toughness and viscoelasticity. Nat. Mater. 12, 932-937 (2013).

32. Kuang, Y., Chen, C., Kirsch, D. \& Hu, L. Thick electrode batteries: principles, opportunities, and challenges. Adv. Energy Mater. 9, 1901457 (2019).

33. Guo, Y., Zheng, K. \& Wan, P. A flexible stretchable hydrogel electrolyte for healable all-in-one configured supercapacitors. Small 14, 1704497 (2018).

34. Yu, J. et al. A high performance stretchable asymmetric fiber-shaped supercapacitor with a core-sheath helical structure. Adv. Energy Mater. 7, 1600976 (2017).

35. Choi, C. et al. Microscopically buckled and macroscopically coiled fibers for ultra-stretchable supercapacitors. Adv. Energy Mater. 7, 1602021 (2017).

36. Chen, C.-R., Qin, H., Cong, H.-P. \& Yu, S.-H. A highly stretchable and realtime healable supercapacitor. Adv. Mater. 31, 1900573 (2019).

37. Chen, D. et al. Self-healing materials for next-generation energy harvesting and storage devices. Adv. Energy Mater. 7, 1700890 (2017).

38. Park, S., Lee, H., Kim, Y.-J. \& Lee, P. S. Fully laser-patterned stretchable microsupercapacitors integrated with soft electronic circuit components. NPG Asia Mater. 10, 959-969 (2018).

39. Zamarayeva, A. M. et al. Flexible and stretchable power sources for wearable electronics. Sci. Adv. 3, e1602051 (2017)

40. Chen, C. et al. All-wood, low tortuosity, aqueous, biodegradable supercapacitors with ultra-high capacitance. Energy Environ. Sci. 10, 538-545 (2017).

41. Dutta, S., Bhaumik, A. \& Wu, K. C.-W. Hierarchically porous carbon derived from polymers and biomass: effect of interconnected pores on energy applications. Energy Environ. Sci. 7, 3574 (2014).

42. $\mathrm{Bi}, \mathrm{Z}$. et al. Biomass-derived porous carbon materials with different dimensions for supercapacitor electrodes: a review. J. Mater. Chem. A 7, 16028 (2019).

\section{Acknowledgements}

We acknowledge the funding support from the National Natural Science Foundation of China (Grants 21922104, 51732011, U1932213, 21571046, and 21503063), the Founda- tion for Innovative Research Groups of the National Natural Science Foundation of China (Grant 21521001), Key Research Program of Frontier Sciences, CAS (Grant No. QYZDJ-SSW-SLH036), the Users with Excellence and Scientific Research Grant of Hefei Science Center of CAS (2015HSC-UE007), the Fundamental Research Funds for the Central Universities (JZ2019HGPB0104, JZ2017HGTB0197, JZ2021HGPA0064), The University Synergy Innovation Program of Anhui Province (GXXT-2019-028), and Anhui Provincial Natural Science Foundation (1908085J05, 1708085MB30).

\section{Author contributions}

H.P.C. and S.H.Y. supervised the project, conceived the idea, designed the experiments, and wrote the paper. H.Q. and P.L. planned and performed the experiments, collected and analyzed the data, and wrote the paper. C.C. helped the electrochemical measurements and analyzed the corresponding data. All authors discussed the results and commented on the manuscript. H.Q. and P.L. contributed equally to this work.

\section{Competing interests}

The authors declare no competing interests.

\section{Additional information}

Supplementary information The online version contains supplementary material available at https://doi.org/10.1038/s41467-021-24568-w.

Correspondence and requests for materials should be addressed to H.-P.C. or S.-H.Y.

Peer review information Nature Communications thanks the anonymous reviewers for their contribution to the peer review of this work.

Reprints and permission information is available at http://www.nature.com/reprints

Publisher's note Springer Nature remains neutral with regard to jurisdictional claims in published maps and institutional affiliations.

(c) (i) Open Access This article is licensed under a Creative Commons Attribution 4.0 International License, which permits use, sharing, adaptation, distribution and reproduction in any medium or format, as long as you give appropriate credit to the original author(s) and the source, provide a link to the Creative Commons license, and indicate if changes were made. The images or other third party material in this article are included in the article's Creative Commons license, unless indicated otherwise in a credit line to the material. If material is not included in the article's Creative Commons license and your intended use is not permitted by statutory regulation or exceeds the permitted use, you will need to obtain permission directly from the copyright holder. To view a copy of this license, visit http://creativecommons.org/ licenses/by/4.0/.

(C) The Author(s) 2021 